\title{
Sur l'ordre des conditions de la coexistence des équations algébriques à plusieurs variables.
}

(Par M. Samuel Roberts à Londres.)

Le beau mémoire de M. Jonquières sur les problèmes de contact des courbes algébriques (ce journal, Tome LXVI, cahier 4) me donne l'occasion d'offrir des résultats auxquels, de mon côté, je suis parvenu indépendamment en faisant usage d'un principe analogue ou plutôt identique. Les questions dont je m'occupe, se rapportent à l'ordre des conditions nécessaires pour que les équations données puissent coexister. Pour démontrer ou pour découvrir plusieurs théorèmes relatifs á des fonctions générales (mais toujours algébriques) on substitue au système donné un système de facteurs linéaires. Par exemple, si $S$ équations, renfermant $k$ variables indépendantes, doivent avoir une solution commune, il est permis d'employer, au lieu de ces équations, $S$ produits composés de facteurs linéaires, en observant que l'ordre des conditions de coexistence ne peut changer de valeur à cause de cette restriction.

L'ordre d'un système de conditions explicites, dont le nombre n'est pas plus grand que le nombre des variables qu'elles renferment, est le produit des degrés des équations de condition. Mais en cas que les conditions se trouvent implicites et qu'on introduise des systèmes étrangers par notre procédé d'élimination, l'ordre du système sera le produit des équations dérivées moins la somme des ordres des systèmes étrangers. Par exemple, si l'on a simultanément

$$
\begin{aligned}
& \text { (1.) } \quad A x+B y=0 \\
& \text { (2.) } \quad A^{\prime} x+B^{\prime} y=0 \\
& \text { (3.) } \quad A^{\prime \prime} x+B^{\prime \prime} y=0
\end{aligned}
$$

et qu'on élimine $x, y$ successivement entre (1.), (2.) et entre (1.), (3.), on aura les conditions $A B^{\prime}-A^{\prime} B=0, A B^{\prime \prime}-A^{\prime \prime} B=0$, en introduisant, cependant, le système étranger $A=0, B=0$. Pour obtenir l'ordre net, il faut soustraire l'ordre de ce dernier système de l'ordre des équations dérivées. Encore, si l'on a simultanément 
(4.) $A x+B y+C z=0$,

(5.) $A^{\prime} x+B^{\prime} y+C^{\prime} z=0$,

(6.) $A^{\prime \prime} x+B^{\prime \prime} y+C^{\prime \prime} z=0$,

(7.) $A^{\prime \prime \prime} x+B^{\prime \prime \prime} y+C^{\prime \prime \prime} z=0$

et qu'on élimine $x, y, z$ entre (4.), (5.), (6.) et entre (4.), (5.), (7.) successivement, le résultat sera d'un ordre connu. Mais, le système

$$
\frac{A}{A^{\prime}}=\frac{B}{B^{\prime}}=\frac{C}{C^{\prime}}
$$

étant étranger, il faut soustraire l'ordre de ce système de celui que nous venons de trouver.

Soit proposé le système linéaire

$$
\left.\begin{array}{c}
A_{1} x+B_{1} y \\
A_{2} x+B_{2} y \\
A_{3} x+B_{3} y \\
\cdots \cdots \\
A_{s} x+B_{s} y
\end{array}\right\}=0,
$$

où $A_{1}, A_{2}, \ldots A_{s}$ sont constants, mais où $B_{1}, B_{2}, \ldots B_{s}$ désignent des fonctions du degré $\alpha$ à l'égard d'autres variables, et qu'on demande l'ordre des conditions de la coexistence du système, c'est-à-dire des conditions nécessaires pour que les équations puissent avoir une racine commune. Dans ce cas, il est aisé de montrer que l'ordre demandé est $\alpha^{s-1}$. En effet, si l'on admet que l'ordre des conditions relatives à un système de $s-1$ équations formées d'une manière semblable, soit $\alpha^{s-2}$ et qu'on considère $1^{\circ}$ le système renfermant les $s-1$ premières équations, $2^{\circ}$ le système renfermant la première et la dernière des équations, on trouvera l'ordre $\alpha^{s-1}$, en prenant les ordres de ces systèmes, savoir $\alpha^{s-2}, \alpha$, et en multipliant l'un des résultats par l'autre. Le système étranger est

$$
\begin{aligned}
& A_{1}=0 \text {, } \\
& B_{1}=0 \text {, } \\
& \left.\begin{array}{c}
A_{2} x+B_{2} y \\
A_{3} x+B_{3} y \\
\ldots . \cdot \\
A_{s-1} x+B_{s-1} y
\end{array}\right\}=0
\end{aligned}
$$

On voit la même chose en éliminant $x, y$ entre la première et la seconde, la 
première et la troisième etc. des équations. En ce cas, la correction généralement effective s'évanouit, et un facteur de l'ordre du seul système étranger sera l'ordre de $\left(A_{1}=0, B_{1}=0\right)$, qui est nul. On voit facilement que la formule est vraie pour $s=2, s=3$, ce qui démontre la proposition.

Supposons maintenant que les lettres $A_{1}, A_{2}, \ldots A_{s}$ désignent des fonctions des degrés $\mu_{1}, \mu_{2}, \ldots \mu_{s}$ par rapport aux variables secondaires, et que les lettres $B_{1}, B_{2}, \ldots B_{s}$ désignent des fonctions des degrés $\mu_{1}+\alpha$, $\mu_{2}+\alpha, \ldots \mu_{s}+\alpha$ par rapport aux mêmes variables. Il est important d'observer que l'ordre des conditions dont nous nous occupons, ne dépend que des degrés des coefficients et qu'il ne dépend point de leurs formes spécifiques. D'après cela, on peut supposer que les coefficients de chaque équation $\boldsymbol{A}_{p} \boldsymbol{x}+\boldsymbol{B}_{p} \boldsymbol{y}=\mathbf{0}$ contiennent un facteur du degré $\mu_{p}$ par rapport aux variables secondaires. Dans cette supposition, l'ordre des conditions deviendra

$$
\text { (A.) } \quad \alpha^{s-1}+\alpha^{s-2} \sum_{1}(\mu)+\alpha^{s-3} \sum_{2}(\mu)+\cdots+\alpha \sum_{s-2}(\mu)+\sum_{s-1}(\mu),
$$

si l'on représente par $\sum_{p}(\mu)$ la somme des produits des quantités $\mu_{1} \mu_{2} \ldots \mu_{s}$ prises $p$ à $p$, en faisant usage du résultat précédent. En effet, on voit que le système peut coexister en cas que $t$ des équations prises arbitrairement coexistent, et que les fonctions s'évanouissent, par lesquelles on multiplie les équations restantes. L'ordre particulier, qu'on obtient dans cette supposition est $\alpha^{t-1} \sum_{s-t}(\mu)$, et l'ordre total s'exprime par $\sum_{t=1}^{t=s}\left[\alpha^{t-1} \sum_{s-t}(\mu)\right]$ si l'on fait usage d'une notation bien connue. Par conséquent, la formule $(A$.) exprime l'ordre cherché dans le cas général.

Si l'on remplace $\mu_{1}$ par $a+\mu_{1}, \mu_{2}$ par $a+\mu_{2}, \mu_{3}$ par $a+\mu_{3}$ et ainsi de suite, je remarque que l'expression $(A$.) peut se mettre sous la forme abrégée

$$
\boldsymbol{D}(\boldsymbol{\Phi}) \text {, }
$$

le symbole $D$ indiquant l'opération

$$
\alpha^{s-1} \frac{\left(\frac{d}{d a}\right)^{s}}{\Pi(s)}+\alpha^{s-2} \frac{\left(\frac{d}{d a}\right)^{s-1}}{\Pi(s-1)}+\alpha^{s-3} \frac{\left(\frac{d}{d a}\right)^{s-2}}{\Pi(s-2)}+\cdots+\alpha \frac{\left(\frac{d}{d a}\right)^{2}}{1.2}+\left(\frac{d}{d a}\right)
$$

et $\Phi$ étant écrit à la place de

$$
a^{s}+a^{s-1} \sum_{1}(\mu)+a^{s-2} \sum_{2}(\mu)+\cdots+a \sum_{s-1}(\mu)+\sum_{s}(\mu) .
$$

Je remarque aussi que le résultat conservera la même forme dans le cas où l'on substitue $P^{\prime}$ au lieu de $\sum_{1}(\mu), P^{\prime \prime}$ au lieu de $\sum_{2}(\mu), P^{\prime \prime \prime}$ au lieu de $\sum_{3}(\mu)$ et ainsi de suite, substitution qu'on fait afin d'exclure des solutions étrangères. 
Soit proposé un système de $s$ équations linéaires, renfermant $k$ variables indépendantes, savoir

$$
\begin{aligned}
& A_{1} x+B_{1} x_{1}+C_{1} x_{2}+\cdots+T_{1} x_{k-1}+V_{1} x_{k}=0, \\
& A_{2} x+B_{2} x_{1}+C_{2} x_{2}+\cdots+T_{2} x_{k-1}+V_{2} x_{k}=0 \text {, } \\
& A_{3} x+B_{3} x_{1}+C_{3} x_{2}+\cdots+T_{3} x_{k-1}+V_{3} x_{k}=0 \text {, } \\
& A_{s} x+B_{s} x_{1}+C_{s} x_{2}+\cdots+T_{s} x_{k-1}+V_{s} x_{k}=0,
\end{aligned}
$$

les ordres des $A_{1}, A_{2}, A_{3}, \ldots A_{s}$, par rapport aux variables secondaires, étant $\mu_{1}, \mu_{2}, \mu_{3}, \ldots \mu_{s} ;$ ceux des $B_{1}, B_{2}, B_{3}, \ldots B_{s}$ étant $\mu_{1}+\alpha_{1}, \mu_{2}+\alpha_{1}$, $\mu_{3}+\alpha_{1}, \ldots \mu_{s}+\alpha_{1} ;$ ceux des $C_{1}, C_{2}, C_{3}, \ldots C_{s}$ étant $\mu_{1}+\alpha_{2}, \mu_{2}+\alpha_{2}$, $u_{3}+\alpha_{2}, \ldots \mu_{s}+\alpha_{2}$; et ainsi de suite, jusqu'à $V_{1}, V_{2}, V_{3}, \ldots V_{s}$, dont les ordres soient $\mu_{1}+\alpha_{k}, \mu_{2}+\alpha_{k}, \mu_{3}+\alpha_{k}, \ldots \mu_{s}+\alpha_{k}$.

En représentant par $S_{p}\left(\alpha_{q}\right)$ la somme des puissances et des produits de $\alpha_{1}, \alpha_{2}, \ldots \alpha_{q}$ d'ordre $p$, je dis que l'ordre des conditions de la coexistence du système proposé est

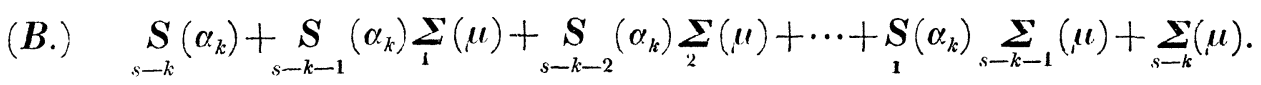

Admettons que cette expression convienne à un tel système renfermant $k-1$ .variables indépendantes, et considérons d'abord le système donné en excluant les termes $V_{1} x_{k}, V_{2} x_{k}, \ldots V_{s} x_{k}$. Dans ce cas on a, par hypothèse, pour l'ordre

$$
\underset{s-k+1}{S}\left(\alpha_{k-1}\right)+\underset{s-k}{S}\left(\alpha_{k-1}\right) \sum_{1}(\mu)+\underset{s-k-1}{S}\left(\alpha_{k-1}\right) \sum_{i}(\mu)+\cdots+\underset{1}{S}\left(\alpha_{k-1}\right) \sum_{s-k}(\mu)+\sum_{s-k+1}(\mu),
$$

expression qui est de la forme

$$
\text { ( } \beta \text {.) } \quad \alpha_{k-1}^{s-k+1}+\alpha_{k-1}^{s-k} P^{\prime}+\alpha_{k-1}^{s-k-1} P^{\prime \prime}+\text { etc. }
$$

Mais, en tenant compte des termes exceptés, le système secondaire dont nous venons de trouver l'ordre à l'égard des variables secondaires, se trouve de l'ordre 1 à l'égard des quantités $x_{k-1}, x_{k}$. Outre cela, il nous faut observer qu'en admettant $x_{k-1}, x_{k}$ dans ce résultat, on admet toujours l'incrément $\alpha_{k}-\alpha_{k-1}$ (il est permis de poser $\alpha_{k}>\alpha_{k-1}$ ) ̀̀ l'ordre des coefficients affectés de $x_{k}$. Par exemple, soit donné le système simple

$$
\left.\begin{array}{l}
A_{1} x+B_{1} x_{1}+C_{1} x_{2} \\
A_{2} x+B_{2} x_{1}+C_{2} x_{2} \\
A_{3} x+B_{3} x_{1}+C_{3} x_{2} \\
A_{4} x+B_{4} x_{1}+C_{4} x_{2}
\end{array}\right\}=0,
$$

Journal für Mathematik Bd. LXVII. Heft 3. 
on obtient le système dérivé

$$
\left.\begin{array}{l}
A_{1}\left(B_{2} x_{1}+C_{2} x_{2}\right)-A_{2}\left(B_{1} x_{1}+C_{1} x_{2}\right) \\
A_{1}\left(B_{3} x_{1}+C_{3} x_{2}\right)-A_{3}\left(B_{1} x_{1}+C_{1} x_{2}\right) \\
A_{1}\left(B_{4} x_{1}+C_{4} x_{2}\right)-A_{4}\left(B_{1} x_{1}+C_{1} x_{2}\right)
\end{array}\right\}=0
$$

dont l'ordre, par rapport aux variables contenues dans les coefficients, est

$$
\left(\alpha_{1}+\mu_{1}+\mu_{2}\right)\left(\alpha_{1}+\mu_{1}+\mu_{3}\right)\left(\alpha_{1}+\mu_{1}+\mu_{4}\right) \text {, c'est-à-dire } \alpha_{1}^{3}+\alpha_{1}^{2} Q^{\prime}+\alpha_{1} Q^{\prime \prime}+Q^{\prime \prime \prime} ;
$$

mais il faut faire une correction de cet ordre, en substituant

$$
\alpha_{1}^{3}+\alpha_{1}^{2} P^{\prime}+\alpha_{1} P^{\prime \prime}+P^{\prime \prime \prime} \text {. }
$$

En cas que cette quantité ne soit pas décomposable en trois facteurs numériques et entiers. trois équations à des indices entiers ne peuvent représenter les conditions, tout de même que deux équations renfermant deux variables indépendantes à des indices entiers ne peuvent représenter un nombre premier de points à moins qu'ils ne se rangent sur une droite. Maintenant, en considèrant le système binaire entre $x_{k-1}, x_{k}$, on a pour l'ordre des conditions de coexistence

$$
D^{\prime}\left(\Phi^{\prime}\right)
$$

en écrivant, pour abréger, $\boldsymbol{\Phi}^{\prime}$ à la place de l'expression ( $\beta$.) et en indiquant par le symbole $D^{\prime}$ l'opération suivante

$$
\begin{gathered}
\left(\alpha_{k}-\alpha_{k-1}\right)^{s-k} \frac{\left(\frac{d}{d \alpha_{k-1}}\right)^{s-k+1}}{\Pi(s-k+1)}+\left(\alpha_{k}-\alpha_{k-1}\right)^{s-k-1} \frac{\left(\frac{d}{d \alpha_{k-1}}\right)^{s-k}}{\Pi(s-k)}+\cdots \\
\quad+\left(\alpha_{k}-\alpha_{k-1}\right) \frac{\left(\frac{d}{d \alpha_{k}}\right)^{2}}{1.2}+\left(\frac{d}{d \alpha_{k}}\right) .
\end{gathered}
$$

Il n'est pas difficile de voir que ce résultat peut se mettre sous la forme (B.). Car on a, d'une manière symbolique:

$$
\begin{aligned}
E^{\left(\alpha_{k}-\alpha_{k-1}\right) \frac{d}{d \alpha_{k-1}}} S_{p}\left(\alpha_{k-1}\right) & =E^{\left(\alpha_{k}-\alpha_{k-1}\right) \frac{d}{d \alpha_{k-1}}}\left\{\alpha_{k-1}^{p}+\alpha_{k-1}^{p-1} S_{1}\left(\alpha_{k-2}\right)+\text { etc. }+S_{p}\left(\alpha_{k-2}\right)\right\} \\
& =\alpha_{k}^{p}+\alpha_{k}^{p-1} S_{1}\left(\alpha_{k-2}\right)+\text { etc. }+S_{p}\left(\alpha_{k-2}\right) ;
\end{aligned}
$$

et il résulte de là qu'on aura

$$
\begin{aligned}
\frac{E^{\left(\alpha_{k}-\alpha_{k-1}\right)} \frac{d}{d \alpha_{k-1}}-1}{\alpha_{k}-\alpha_{k-1}} S_{p}\left(\alpha_{k-1}\right) & =\frac{\alpha_{k}^{p}-\alpha_{k-1}^{p}}{\alpha_{k}-\alpha_{k-1}}+\frac{\alpha_{k}^{p-1}-\alpha_{k-1}^{p-1}}{\alpha_{k}-\alpha_{k-1}} S_{1}\left(\alpha_{k-2}\right)+\text { etc. }+S_{p-1}\left(\alpha_{k-2}\right) \\
& =S_{p-1}\left(\alpha_{k}\right)
\end{aligned}
$$

en regardant $\alpha_{k}-\alpha_{k-1}$ comme constant. 
Mais nous avons déjà montré que la formule $(B$.) convient à un système binaire; ce qui complète la démonstration.

M. Salmon est parvenu, par induction, au même résultat, en l'établissant rigoureusement dans les cas où $\left.s=k+2, s=k+3^{*}\right)$. Au reste la formule est vraie, évidt... inent, pour $s=k+1$.

Désignons maintenant par

$$
P_{v}\left(A x+B x_{1}+C x_{2}+\cdots T x_{k-1}+V x_{k}\right)
$$

un produit de $v$ facteurs d'une forme semblable à celle qui se trouve représentée. Si l'on a le système simultané

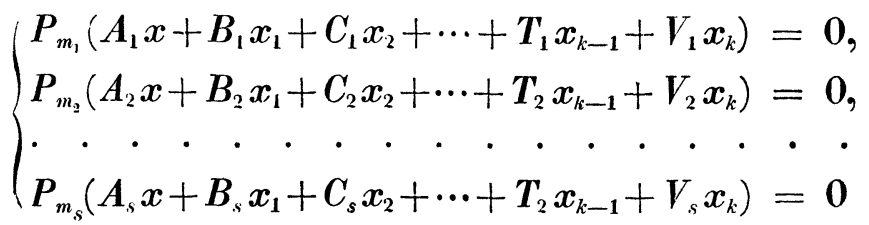

les lettres $A_{1}, A_{2}, \ldots ; B_{1}, B_{2}, \ldots$ etc. conservant leurs significations précédentes, et qu'on propose de trouver l'ordre des conditions nécessaires pour que ces équations puissent avoir une solution commune.

On a, tout d'un coup, pour l'ordre cherché

$$
m_{1} m_{2} \ldots m_{s} \sum_{t=1}^{t=s-k}\left(\underset{t}{S}\left(\alpha_{k}\right) \underset{s-k-t}{\sum}(\mu)\right) \text {. }
$$

Car on peut former $m_{1} m_{2} \ldots m_{s}$ systèmes linéaires distincts en prenant toujours un facteur de chacune des équations. Or, si l'on développe les produits, on obtiendra des fonctions dans lesquelles les coefficients des $x^{m_{1}}, x^{m_{2}}, \ldots x^{m_{s}}$ seront des ordres $m_{1} \mu_{1}, m_{2} \mu_{2}, \ldots m_{s} \mu_{s}$ respectivement, ceux des $x^{m_{1}-1} x_{1}$, $x^{m_{2}-1} x_{1}, \ldots x^{m_{s}-1} x_{1}$ seront des ordres $m_{1} u_{1}+\alpha_{1}, m_{2} \mu_{2}+\alpha_{1}, \ldots m_{s} u_{s}+\alpha_{1}$, et ainsi de suite.

Donc, en mettant $\lambda_{1}$ au lieu de $m_{1} \mu_{1}, \lambda_{2}$ au lieu de $m_{2} \mu_{2}$, etc., je dis qu'on aura pour l'ordre propre à un système général la valeur

$$
\text { (C.) } \quad m_{1} m_{2} \ldots m_{s} \sum_{t=1}^{t=s-k}\left(S\left(\alpha_{k}\right) \sum_{s-k-t}\left(\frac{\lambda}{m}\right)\right)
$$

ou, ce qui reviest au même,

$$
\left\{\begin{array}{c}
\underset{s-k}{S}\left(\alpha_{k}\right) m_{1} m_{2} \ldots m_{s}+\underset{s-k-1}{S}\left(\alpha_{k}\right) \Sigma \mu_{1} m_{2} \ldots m_{s}+\underset{s-k-2}{S}\left(\alpha_{k}\right) \Sigma \mu_{1} \mu_{2} m_{3} \ldots m_{s}+\ldots \\
+S\left(\alpha_{k}\right) \Sigma \mu_{1} \mu_{2} \mu_{s-k-1} m_{s-k} \ldots m_{s}+\sum \mu_{1} \ldots \mu_{s-k} m_{s-k+1} \ldots m_{s}
\end{array}\right.
$$

*) Voyez ses "Lessons introductory to the modern Higher Algebra“, édition seconde, p. 229. 
En cas que $\frac{\lambda_{p}}{m_{p}}$ soit un nombre fractionnaire, on peut observer qu'on obtiendra le mème résultat en distribuant l'ordre $\lambda_{p}$ entre les facteurs d'une manière quelconque afin d'éviter les expressions fractionnaires. Il est nécessaire, seulement, que les incréments $\alpha_{1}, \alpha_{2}, \alpha_{3}$, etc. demeurent les mêmes dans tous les facteurs.

Appliquons tout de suite la formule $\left(C^{\prime}\right.$.) à un exemple. On propose de trouver l'ordre des conditions nécessaires pour qu'une courbe algébrique puisse posséder un point de rebroussement.

Soit $u_{r}=0$ l'équation de la courbe, $u_{r}$ étant une fonction du degré $r$, homogène à l'égard des coordonnées trilinéaires $x, y, z$. Supposons, de plus, que le coefficient du terme affecté de $x^{r}$ contienne d'autres variables au degré $\varrho$, et que l'ordre du coefficient d'un terme quelconque éprouve les incréments $\alpha_{1}, \alpha_{2}$ toutes les fois que le terme est affecté de $y, z$ respectivement, en sorte que le coefficient de $x^{1-1} y$ est de l'ordre $\varrho+\alpha_{1}$, celui de $x^{r-3} y z^{2}$ est de l'ordre $0+\alpha_{1}+2 \alpha_{2}$, et ainsi de suite.

Il faudra que les coordonnées d'un point de rebroussement satisfassent aux équations

$$
\text { (r.) } \quad \frac{d u_{r}}{d x}=\frac{d u_{r}}{d y}=\frac{d u_{r}}{d z}=\frac{d^{2} u_{r}}{d x^{2}} \frac{d^{2} u_{r}}{d y^{2}}-\left(\frac{d^{2} u_{r}}{d x d y}\right)^{2}=0 .
$$

Ainsi, mettons dans la formule $\left(C^{\prime}\right.$.) $s=4, k=2, r-1$ au lieu de $m_{1}, m_{2}$, $m_{3}$ respectivement, $2(r-2)$ au lieu de $m_{4} ; \varrho, \varrho+\alpha_{1}, \varrho+\alpha_{2}, 2 \varrho+2 \alpha_{1}$ au lieu de $\mu_{1}, \mu_{2}, \mu_{3}, \mu_{4}$. De cette manière on obtient l'ordre des conditions de coexistence sous la forme

$$
\left\{\begin{array}{c}
2(r-1)^{2}\left(\varrho+\alpha_{1}\right)\left(3 \varrho+\alpha_{1}+\alpha_{2}\right)+2(r-1)(r-2)\left(3 \varrho^{2}+2\left(\alpha_{1}+\alpha_{2}\right) \varrho+\alpha_{1} \alpha_{2}\right) \\
+2\left(\alpha_{1}+\alpha_{2}\right)\left\{(r-1)^{3}\left(\varrho+\alpha_{1}\right)+(r-1)^{2}(r-2)\left(3 \varrho+\alpha_{1}+\alpha_{2}\right)\right\}+2(r-1)^{3}(r-2)\left(\alpha_{1}^{2}+\alpha_{2}^{2}+\alpha_{1} \alpha_{2}\right) .
\end{array}\right.
$$

Mais il faut observer que les coordonnées d'un point double situé sur la droite $(z=0)$ satisferont aux conditions $\left(\gamma_{\circ}\right)$. Par conséquent, on doit soustraire l'ordre qui appartient à ce cas.

On a, par rapport à un tel point double,

$$
\frac{d u_{r}}{d x}=\frac{d u_{r}}{d y}=\frac{d u_{r}}{d z}=z=0
$$

L'ordre de ce système est par $\left(C^{\prime}\right.$.)

(b.) $\quad(r-1)\left(3 \varrho^{2}+2\left(\alpha_{1}+\alpha_{2}\right) \varrho+\alpha_{1} \alpha_{2}\right)+(r-1)^{2}\left(3 \varrho+\alpha_{1}+\alpha_{2}\right) \alpha_{1}+(r-1)^{3} \alpha_{1}^{2}$.

Nous devons prendre le double de ce résultat parce qu'il se rapporte à un point double. Enfin, on a 


$$
\text { (c.) }\left\{\begin{aligned}
(a .)-2(b .) & =12(r-1)(r-2) \varphi^{2}+8 r(r-1)(r-2)\left(\alpha_{1}+\alpha_{2}\right) \varrho \\
& +2 r(r-1)(r-2)(r+1) \alpha_{1} \alpha_{2}+2 r(r-1)^{2}(r-2)\left(\alpha_{1}^{2}+\alpha_{2}^{2}\right),
\end{aligned}\right.
$$

ce qui est l'ordre cherché.

Pour donner un autre exemple de l'application de la formule générale que nous venons d'établir, on propose de trouver l'ordre des conditions nécessaires pour qu'une surface algébrique puisse posséder un point double tel que le cône tangent soit composé de deux plans (c'est-àdire le point double doit être bi-planaire).

Soit $V_{r}=0$ l'équation de la surface, $V_{r}$ étant une fonction du degré $r$, homogène à l'égard des coordonnées quadri-planaires $x, y, z, w$. On suppose que le coefficient de $x^{r}$ est de l'ordre $\varrho$ à l'égard d'autres variables et que les incréments des ordres des coefficients par rapport à $y, z, w$ sont $\alpha_{1}$, $\alpha_{2}, \alpha_{3}$ respectivement, en sorte que l'ordre, par exemple, du coefficient de $x^{r-4} y z^{2} w$ soit $\varrho+\alpha_{1}+2 \alpha_{2}+\alpha_{3}$. Les conditions auxquelles les coordonnées d'un tel point double bi-planaire doivent satisfaire, sont

$$
\text { (ঠ.) } \quad \frac{d V_{r}}{d x}=\frac{d V_{r}}{d y}=\frac{d V_{r}}{d z}=\frac{d V_{r}}{d w}=\boldsymbol{H}=\mathbf{0},
$$

la lettre $\boldsymbol{H}$ étant écrite pour le déterminant

$$
\left|\begin{array}{lll}
\frac{d^{2} V_{r}}{d x^{2}}, & \frac{d^{2} V_{r}}{d x d y}, & \frac{d^{2} V_{r}}{d x d z} \\
\frac{d^{2} V_{r}}{d x d y}, & \frac{d^{2} V_{r}}{d y^{2}}, & \frac{d^{2} V_{r}}{d y d z} \\
\frac{d^{2} V_{r}}{d x d z}, & \frac{d^{2} V_{r}}{d y d z}, & \frac{d^{2} V_{r}}{d z^{2}}
\end{array}\right| .
$$

Par conséquent, nous devons mettre dans la formule $\left(C^{\prime}.\right) s=5, k=3, r-1$ au lieu de $m_{1}, m_{2}, m_{3}, m_{4}$ respectivement, $3(r-2)$ au lieu de $m_{5}, \varrho, \varrho+\alpha_{1}$, $\varrho+\alpha_{2}, \varrho+\alpha_{3}, 3 \varrho+2 \alpha_{1}+2 \alpha_{2}$ au lieu de $\mu_{1}, \mu_{2}, \mu_{3}, \mu_{4}, \mu_{5}$. Il résulte de là que l'ordre du système se met sous la forme

$\left(\boldsymbol{a}^{\prime}\right) . \quad\left\{\begin{array}{c}+(r-1)^{3}\left(4 \varrho+\alpha_{1}+\alpha_{2}+\alpha_{3}\right)\left(3 \varrho+2 \alpha_{1}+2 \alpha_{2}\right) \\ +\left(\alpha_{1}+\alpha_{2}+\alpha_{3}\right)\left\{(r-1)^{4}\left(3 \varrho+2 \alpha_{1}+2 \alpha_{2}\right)+3(r-1)^{3}(r-2)\left(4 \varrho+\alpha_{1}+\alpha_{2}+\alpha_{3}\right)\right\} \\ +3(r-1)^{4}(r-2)\left(\alpha_{1}^{2}+\alpha_{2}^{2}+\alpha_{3}^{2}+\alpha_{1} \alpha_{2}+\alpha_{1} \alpha_{3}+\alpha_{2} \alpha_{3}\right) .\end{array}\right.$

Mais il faut observer que les coordonnées d'un point double situé dans le plan $(u=0)$ satisferont aux conditions $\left(\delta_{0}\right)$. Par conséquent on doit soustraire du résultat obtenu ci-dessus le double de l'ordre propre au système 


$$
\frac{d V_{r}}{d x}=\frac{d V_{r}}{d y}=\frac{d V_{r}}{d z}=\frac{d V_{r}}{d w}=w=0 .
$$

En meltant dans la formule $\left(C^{\prime}\right.$.) $s=4, k=2$, et en substituant $r-1$ à la place de $m_{1}, m_{2}, m_{3}, m_{4}$ respectivement, $\varrho, \varrho+\alpha_{1}, \varrho+\alpha_{2}, \varrho+\alpha_{3}$ à la place de $\mu_{1}, \mu_{2}, \mu_{3}, \mu_{4}$ respectivement, on aura

$$
\left(b^{\prime} .\right) \quad\left\{\begin{array}{c}
(r-1)^{2}\left\{6 \varrho^{2}+3\left(\alpha_{1}+\alpha_{2}+\alpha_{3}\right) \varrho+\alpha_{1} \alpha_{2}+\alpha_{1} \alpha_{3}+\alpha_{2} \alpha_{3}\right\} \\
+(r-1)^{3}\left(\alpha_{1}+\alpha_{2}\right)\left(4 \varrho+\alpha_{1}+\alpha_{2}+\alpha_{3}\right)+(r-1)^{4}\left(\alpha_{1}^{2}+\alpha_{2}^{2}+\alpha_{3}^{2}\right) .
\end{array}\right.
$$

Enfin, on trouve

$$
\left(c^{\prime} .\right)\left\{\begin{array}{l}
\left(a^{\prime} .\right)-2\left(b^{\prime} .\right)=30(r-1)^{2}(r-2) \varrho^{2}+15 r(r-1)^{2}(r-2)\left(\alpha_{1}+\alpha_{2}+\alpha_{3}\right) \\
+r(r-1)^{2}(r-2)(3 r+2)\left(\alpha_{1} \alpha_{2}+\alpha_{1} \alpha_{3}+\alpha_{2} \alpha_{3}\right)+3 r(r-1)^{3}(r-2)\left(\alpha_{1}^{2}+\alpha_{2}^{2}+\alpha_{3}^{2}\right)
\end{array}\right.
$$

ce qui est l'ordre cherché.

Le même procédé que nous venons d'employer peut s'ètendre au cas où les systèmes d'équations doivent avoir deux ou plusieurs solutions distinctes communes. Prenons le système

$$
\begin{aligned}
& P_{m_{1}}\left(A_{1} x+B_{1} x_{1}+C_{1} x_{2}\right)=0, \\
& P_{m_{3}}\left(A_{2} x+B_{2} x_{1}+C_{2} x_{2}\right)=0, \\
& P_{m_{3}}\left(A_{3} x+B_{3} x_{1}+C_{3} x_{2}\right)=0,
\end{aligned}
$$

en supposant que $A_{1}, B_{1}, C_{1}$ désignent des fonctions des degrés $\frac{\mu_{1}}{m_{1}}, \frac{\mu_{1}}{m_{1}}+\alpha_{1}$, $\frac{\mu_{1}}{m_{1}}+\alpha_{2}$, que $A_{2}, B_{2}, C_{2}$ désignent des fonctions des degrés $\frac{\mu_{1}}{m_{1}}, \frac{\mu_{1}}{m_{1}}+\alpha_{1}$, $\frac{\mu_{2}}{m_{2}}+\alpha_{2}$, et que $A_{3}, B_{3}, C_{3}$ désignent des fonctions des degrés $\frac{\mu_{3}}{m_{3}}, \frac{\mu_{3}}{m_{3}}+\alpha_{1}$, $\frac{\mu_{3}}{m_{3}}+\alpha_{2}$.

Considérons, premièrement, un système qui contient deux facteurs quelconques de chacune des équations, savoir:

$$
\begin{aligned}
& K_{1} \cdot L_{1}=0, \\
& K_{2} \cdot L_{2}=0, \\
& K_{3} \cdot L_{3}=0
\end{aligned}
$$

en représentant par les lettres les facteurs respectifs. Ce système nous donne l'ordre

$$
4\left(\frac{\mu_{1}}{m_{1}}+\frac{\mu_{2}}{m_{2}}+\frac{\mu_{3}}{m_{3}}+\alpha_{1}+\alpha_{2}\right)^{2} \text { ou } 4 \varphi^{2} .
$$

Car on a, par exemple, les deux systèmes

$$
\begin{aligned}
& K_{1} \\
& K_{2} \\
& K_{3}
\end{aligned}\left|=0, \quad \begin{array}{l}
L_{1} \\
L_{2} \\
L_{3}
\end{array}\right|=0
$$

qui nous donnent l'ordre $\varphi^{2}$. Il $\mathrm{y}$ a quatre systèmes analogues. 
Considérons, en second lieu, un système qui contient deux facteurs de la première équation, deux facteurs de la seconde et un facteur de la dernière, savoir

$$
\begin{aligned}
& \boldsymbol{K}_{1} \cdot L_{1}=\mathbf{0}, \\
& \boldsymbol{K}_{2} \cdot L_{2}=\mathbf{0}, \\
& \boldsymbol{K}_{3}=\mathbf{0}
\end{aligned}
$$

d'où l'on tire, de la même manière que ci-dessus l'ordre $=2 \varphi^{2}$.

Considérons, enfin, un système qui contient deux facteurs de la première équation et un facteur de chacune des autres, savoir

$$
\begin{aligned}
& \boldsymbol{K}_{1} \cdot L_{1}=\mathbf{0}, \\
& \boldsymbol{K}_{2}=0, \\
& \boldsymbol{K}_{3}=0,
\end{aligned}
$$

équations d'où nous tirons l'ordre $\left(\frac{\mu_{2}}{m_{2}}\right)^{2}+\left(\frac{\mu_{3}}{m_{3}}\right)^{2}+\frac{\mu_{2} \mu_{3}}{m_{2} m_{3}}+\left(\alpha_{1}+\alpha_{2}\right)\left(\frac{\mu_{2}}{m_{2}}+\frac{\mu_{3}}{m_{3}}\right)+\alpha_{1} \alpha_{2}$ en faisant coincider $K_{2}, K_{3}$. Il s'agit maintenant de déterminer les multiplicateurs numériques des ordres que nous venons de trouver. On obtient, par le calcul des combinaisons, les expressions

$\frac{m_{1} \cdot m_{2} \cdot m_{3}\left(m_{1}-1\right)\left(m_{2}-1\right)\left(m_{3}-1\right)}{(1.2)^{3}}, \frac{m_{1} \cdot m_{2} \cdot m_{3}\left(m_{1}-1\right)\left(m_{2}-2\right)}{(1.2)^{2}}, \frac{m_{1} m_{2} m_{3}\left(m_{1}-1\right)}{1.2}$.

Donc, en formant les termes symétriques, on aura, pour l'ordre des conditions à remplir pour que le système donné puisse avoir deux solutions distinctes communes (mais peut-être infiniment voisines), l'expression suivante

(D.)

$$
\left\{\begin{aligned}
\frac{m_{1} m_{2} m_{3}}{2}\{ & {\left[\left(m_{1}-1\right)\left(m_{2}-1\right)\left(m_{3}-1\right)+\Sigma\left(m_{1}-1\right)\left(m_{2}-1\right)\right]\left(\frac{\mu_{1}}{m_{1}}+\frac{\mu_{2}}{m_{2}}+\frac{\mu_{3}}{m_{3}}+\alpha_{1}+\alpha_{2}\right)^{2} } \\
& \left.+\Sigma\left(m_{1}-1\right)\left(\left(\frac{\mu_{2}}{m_{2}}\right)^{2}+\left(\frac{\mu_{3}}{m_{3}}\right)^{2}+\frac{\mu_{2} \mu_{3}}{m_{2} m_{3}}+\left(\alpha_{1}+\alpha_{2}\right)\left(\frac{\mu_{2}}{m_{2}}+\frac{\mu_{3}}{m_{3}}\right)+\alpha_{1} \alpha_{2}\right)\right\} .
\end{aligned}\right.
$$

Je dis donc que ce résultat n'est autre chose que l'ordre des conditions propres à un système de trois équations générales des degrés $m_{1}, m_{2}, m_{3}$, les coefficients de $x^{m_{1}}, x^{m_{2}}, x^{m_{3}}$ étant respectivement des ordres $\mu_{1}, \mu_{2}, \mu_{3}$, ceux de $x^{m_{1}-1} x_{1}, x^{m_{2}-1} x_{1}, x^{m_{3}-1} x_{1}$ étant respectivement des ordres $\mu_{1}+\alpha_{1}, \mu_{2}+\alpha_{1}$, $\mu_{3}+\alpha_{1}$, et ainsi de suite; en d'autres termes, c'est l'ordre des conditions à remplir pour que trois courbes planes puissent avoir deux intersections communes.

La formule s'accorde avec celle que M. Salmon a trouvée en suivant une route différente ${ }^{*}$ ).

*) Voyez ses „Lessons introductory to the modern Higher Algebra“, édition seconde, p. 238 . Dans une note vers la fin de ce livre l'auteur a bien voulu faire mention de la méthode de ce mémoire. 
Les mêmes procédés nous conduisent à une formule plus générale, applicable au système $\left(I^{\prime}\right.$.) en faisant $s=k+1$. Il n'est pas nécessaire d'entrer dans les détails de la méthode qui ne diffère pas de la précédente. L'expression deviendra

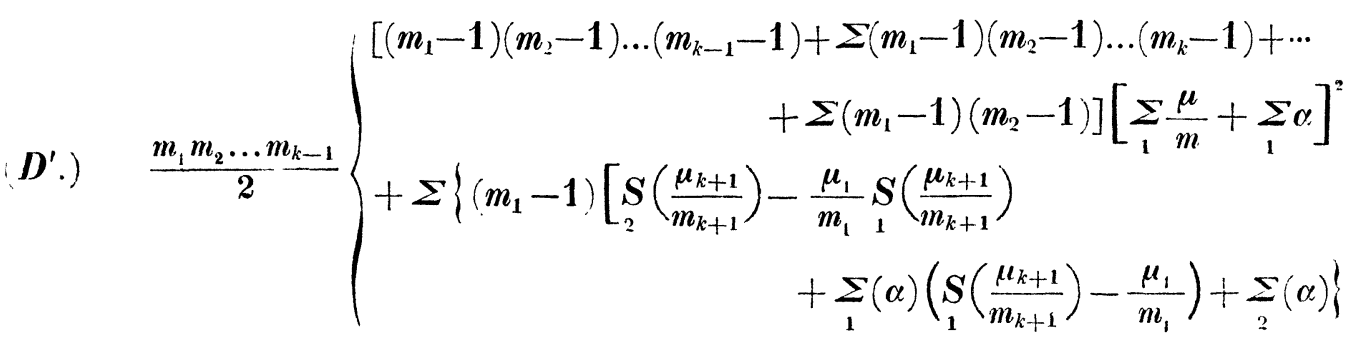

Proposons-nous d'appliquer la formule $(\boldsymbol{D}$.) à la détermination de l'ordre des conditions nécessaires pour qu'une courbe plane puisse avoir deux points doubles. Les coordonnées d'un tel point satisferont aux équations

$$
\frac{d U_{r}}{d x}=\frac{d U_{r}}{d y}=\frac{d U_{r}}{d z}=0
$$

si nous conservons notre notation précédente.

Mettons, donc, dans la formule (D.) obtenu ci-dessus $r-1$ au lieu de $m_{1}, m_{2}, m_{3}$ respectivement, $\varrho, \varrho+\alpha_{1}, \varrho+\alpha_{2}$, au lieu de $\mu_{1}, \mu_{2}, \mu_{3}$ respectivement. De là on obtient l'ordre

$$
\frac{(r-1)(r-2)^{2}(r+1)}{2}\left\{3 \varrho+r\left(\alpha_{1}+\alpha_{2}\right)\right\}^{2}
$$

$+\frac{(r-1)(r-2)}{2}\left\{9 \varrho^{2}+6 r\left(\alpha_{1}+\alpha_{2}\right) \varrho+3(r-1)^{2}\left(\alpha_{1} \alpha_{2}\right)+2(r-1)\left(\alpha_{1}+\alpha_{2}\right)^{2}+2 \alpha_{1}^{2}+2 \alpha_{2}^{2}+\alpha_{1} \alpha_{2}\right\}$.

Mais il faut soustraire de ce résultat l'ordre propre à un point de rebroussement, savoir la quantité (c.). Enfin, il résulte que l'ordre demandé est

$$
-\frac{(r-1)(r-2)}{2}\left[15 \varrho^{2}+10 r\left(\alpha_{1}+\alpha_{2}\right) \varrho+r(r+6) \alpha_{1} \alpha_{2}+2 r(2 r-3)\left(\alpha_{1}^{2}+\alpha_{2}^{2}\right)\right] .
$$

Proposons-nous de trouver l'ordre des conditions nécessaires pour qu'une surface algébrique $V_{r}=0$ puisse avoir deux points doubles. Il faut mettre

$$
\frac{d V_{r}}{d x}=\frac{d V_{r}}{d y}=\frac{d V_{r}}{d z}=\frac{d V_{r}}{d w}=0 .
$$

En posant, donc, dans la formule $\left(D^{\prime}\right.$.) $k=3$, et en substituant $r-1$ au lieu de $m_{1}, m_{2}, m_{3}, m_{4}$ respectivement, $\varrho, \varrho+\alpha_{1}, \varrho+\alpha_{2}, \varrho+\alpha_{3}$ au lieu de $\mu_{1}$, $\mu_{2}, \mu_{3}, \mu_{4}$ on a

$\frac{(r-1)^{2}(r-2)^{2}\left(r^{2}+2\right)}{2}\left\{4 \varrho+\left(\alpha_{1}+\alpha_{2}+\alpha_{3}\right) r\right\}^{2}+\frac{(r-1)^{2}(r-2)^{2}}{2}\left\{24 \varrho^{2}+12 \varrho\left(\alpha_{1}+\alpha_{2}+\alpha_{3}\right)\right.$ $+3\left(\alpha_{1}^{2}+\alpha_{2}^{2}+\alpha_{3}^{2}\right)+2\left(\alpha_{1} \alpha_{2}+\alpha_{1} \alpha_{3}+\alpha_{2} \alpha_{3}\right)+(r-1)\left(12 \rho+3\left(\alpha_{1}+\alpha_{2}+\alpha_{3}\right)+4(r-1)^{2}\left(\alpha_{1} \alpha_{2}+\alpha_{1} \alpha_{3}+\alpha_{2} \alpha_{3}\right)\right\}$. 
En retranchant de ce résultat l'ordre $\left(c^{\prime}\right.$.) qui se rapporte à un point double biplanaire, on obtient

$$
\begin{gathered}
\frac{(r-1)^{2}(r-2)^{2}\left(r^{2}+2\right)}{2}\left(4 \varrho+r\left(\alpha_{1}+\alpha_{2}+\alpha_{3}\right)\right)^{2}-\frac{(r-1)^{2}(r-2)}{2}\left\{36 \varrho^{2}+18 r\left(\alpha_{1}+\alpha_{2}+\alpha_{3}\right) \varrho\right. \\
\left.+3 r(2 r-3)\left(\alpha_{1}^{2}+\alpha_{2}^{2}+\alpha_{3}^{2}\right)+2 r(2 r+3)\left(\alpha_{1} \alpha_{2}+\alpha_{1} \alpha_{3}+\alpha_{2} \alpha_{3}\right)\right\}
\end{gathered}
$$

ce qui est l'ordre cherché.

On pourrait étendre la méthode à plusieurs problèmes du même genre. En suivant la même voie je suis parvenu à l'expression de l'ordre des conditions nécessaires pour que quatre courbes planes puissent avoir deux intersections communes. A présent je ne veux donner que l'expression de l'ordre des conditions nécessaires pour que trois courbes planes puissent avoir trois points communs. En conservant la notation que nous avons employée cidessus, l'ordre s'exprime comme il suit

$$
\begin{aligned}
& m_{1} m_{2} m_{3}\left\{\begin{array}{l}
\frac{\left(m_{1}-1\right)\left(m_{1}-2\right)\left(m_{2}-1\right)\left(m_{2}-2\right)\left(m_{3}-1\right)\left(m_{3}-2\right)}{2.3} \\
+\sum \frac{\left(m_{1}-1\right)\left(m_{2}-1\right)\left(m_{2}-2\right)\left(m_{3}-1\right)\left(m_{3}-2\right)}{2} \\
+3 \Sigma \frac{\left(m_{1}-1\right)\left(m_{1}-2\right)\left(m_{2}-1\right)\left(m_{2}-2\right)}{2.3} \\
+\sum \frac{\left(m_{1}-1\right)\left(m_{1}-2\right)\left(m_{2}-1\right)}{2} \\
+\sum \frac{\left(m_{1}-1\right)\left(m_{2}-1\right)}{2.3}+4\left(m_{1}-1\right)\left(m_{2}-1\right)\left(m_{3}-1\right)
\end{array} \mid\left(\frac{\mu_{1}}{m_{1}}+\frac{\mu_{2}}{m_{2}}+\frac{\mu_{3}}{m_{3}}+\alpha_{1}+\alpha_{2}\right)^{3}\right. \\
& -m_{1} m_{2} m_{3} \Sigma\left(\frac{\left(m_{1}-1\right)\left(m_{1}-2\right)\left(m_{2}-1\right)\left(m_{3}-1\right)}{2}+\frac{\left(m_{1}-1\right)\left(m_{1}-2\right)\left(m_{2}-1\right)}{2}+\frac{\left(m_{1}-1\right)\left(m_{2}-1\right)}{2}\right. \\
& \left.+\left(m_{1}-1\right)\left(m_{2}-1\right)\left(m_{3}-1\right)\right)\left(\frac{\mu_{1}}{m_{1}}+\frac{\mu_{2}}{m_{2}}+\frac{\mu_{3}}{m_{3}}+\alpha_{1}+\alpha_{2}\right)\left(\frac{\mu_{1}^{2}}{m_{1}^{2}}+2 \frac{\mu_{1}}{m_{1}}\left(\frac{\mu_{2}}{m_{2}}+\frac{\mu_{3}}{m_{3}}\right)\right. \\
& \left.+\frac{\mu_{2} \mu_{3}}{m_{2} m_{3}}+\left(\alpha_{1}+\alpha_{2}\right)\left(2 \frac{\mu_{1}}{m_{1}}+\frac{\mu_{2}}{m_{2}}+\frac{\mu_{3}}{m_{3}}\right)+\alpha_{1}^{2}+\alpha_{2}^{2}+\alpha_{1} \alpha_{2}\right) \\
& +m_{1} m_{2} m_{3} \Sigma \frac{\left(m_{1}-1\right)\left(m_{1}-2\right)}{3}\left(\frac{\mu_{1}^{3}}{m_{1}^{3}}+3 \frac{\mu_{1}^{2} \mu_{3}}{m_{1}^{2} m_{3}}+\left(\alpha_{1}+\alpha_{2}\right)\left(3 \frac{\mu_{1}^{2}}{m_{1}^{2}}+3 \frac{\mu_{1} \mu_{3}}{m_{1} m_{3}}\right)\right. \\
& \left.+\left(\alpha_{1}^{2}+\alpha_{2}^{2}+\alpha_{1} \alpha_{2}\right)\left(3 \frac{\mu_{1}}{m_{1}}+\frac{\mu_{3}}{m_{3}}\right)+\left(\alpha_{1}^{3}+\alpha_{2}^{3}+\alpha_{1}^{2} \alpha_{2}+\alpha_{1} \alpha_{2}^{2}\right)\right) \\
& +m_{1} m_{2} m_{3} \Sigma\left(m_{1}-1\right)\left(m_{2}-1\right) \frac{\mu_{3}}{m_{3}}\left(\frac{\mu_{3}}{m_{3}}+\alpha_{1}\right)\left(\frac{\mu_{3}}{m_{3}}+\alpha_{2}\right) \text {. }
\end{aligned}
$$

Journal für Mathematik Bd. LXVII. Heft 3. 
L'établissement de ce résultat n'est pas très-difficile, mais il demande nécessairement un procédé assez long, vu qu'il faut considérer sept espèces de systèmes élémentaires. En représentant des facteurs hypothétiques des équations par des lettres, on peut désigner les systèmes élémentaires comme il suit
A.B.C
A.B.C
A.B.C
A.B.C
A. B. C
A. B. C
A.B
D.E.F
D.E.F
D.E.F
D. E
D.E
$D$
D. $E$
G.H.K G.H
G
G.H
G
G
$\boldsymbol{G}$

De cette manière, le problème se réduit à la détermination des ordres propres à des systèmes linéaires.

Il est évident qu'on peut résoudre beaucoup de problèmes particuliers au moyen des formules générales que nous venons de trouver.

Londres, 14. Février 1867. 